Authors' Contribution:

A Study Design

B Data Collection

C Statistical Analysis

D Data Interpretation

E Manuscript Preparation

F Literature Search

G Funds Collection

\section{Side to side differences in patellar tendon kinetics of the support limb during maximal instep soccer kicking}

\author{
Jonathan Kenneth Sinclair ${ }^{A B C D E F}$, Hannah Shore ${ }^{A B C D}$, Stephen Atkins ${ }^{A B C D}$, \\ Sarah Jane Hobbs ${ }^{\text {ABCDEF }}$
}

Centre for Applied Sport and Exercise Sciences, School of Sport Tourism and Outdoors, University of Central Lancashire, UK

\title{
abstract
}

Background Kicking actions have been implicated in the aetiology of soccer injuries, and the unilateral nature of kicking may influence this. The aim of the current investigation was to determine whether soccer players exhibit bilateral differences in support limb patellar tendon loads.

Material/Methods

Results

Conclusions

Key words
Fifteen male soccer players were examined whilst kicking a stationary soccer ball with both their dominant and non-dominant feet. Patellar tendon kinetics were obtained from each limb and then contrasted using paired samples t-tests.

Significant increases in patellar tendon kinetics were found when using the non-dominant limb.

The findings from the current investigation have clinical significance and support the notion that kicking with the non-dominant limb may be associated with increased risk for patellar tendon injury aetiology.

biomechanics, knee, soccer, tendon

\section{article details}

Article statistics

Full-text PDF:

Copyright

Indexation:

Funding:

Conflict of interest: Corresponding author:

Open Access License:
Word count: 3,281; Tables: 1; Figures: 2; References: 27

Received: February 2015; Accepted: September 2015; Published: December 2015

http://www.balticsportscience.com

(c) Gdansk University of Physical Education and Sport, Poland

AGRO, Celdes, CNKI Scholar (China National Knowledge Infrastructure), CNPIEC, De Gruyter - IBR (International Bibliography of Reviews of Scholarly Literature in the Humanities and Social Sciences), De Gruyter - IBZ (International Bibliography of Periodical Literature in the Humanities and Social Sciences), DOAJ, EBSCO - Centra \& Eastern European Academic Source, EBSCO - SPORTDiscus, EBSCO Discovery Service, Google Scholar, Index Copernicus, J-Gate, Naviga (Softweco, Primo Central (ExLibris), ProQuest - Family Health, ProQuest - Health \& Medical Complete, ProQuest - Illustrata: Health Sciences, ProQuest - Nursing \& Allied Health Source, Summon (Serials Solutions/ProQuest, TDOne (TDNet), Ulrich's Periodicals Directory/ulrichsweb, WorldCat (OCLC)

This research received no specific grant from any funding agency in the public, commercial, or not-for-profit sectors.

Authors have declared that no competing interest exists.

Dr. Jonathan Sinclair, Division of Sport, Exercise and Nutritional Sciences, School of Sport Tourism and Outdoors, University of Central Lancashire, Preston, Lancashire, PR1 2HE. e-mail: Jksinclair@uclan.ac.uk

This is an open access article distributed under the terms of the Creative Commons Attribution-Non-commercial 4.0 International (http://creativecommons.org/licenses/by-nc/4.0/), which permits use, distribution, and reproduction in any medium, provided the original work is properly cited, the use is non-commercial and is otherwise in compliance with the license. 


\section{INTRODUCTION}

Instep kicking is one of the skills that is central to effective soccer performance [1] and is therefore the most frequently investigated movement in soccer specific research [2]. Soccer has a relatively high incidence of injury in relation to other dynamic sports [3]. Injury frequency has been shown to be up to $29.1 \mathrm{in}$ juries per 1000 hours of either playing time or training [4]. Importantly, clinical analyses investigating the aetiology of soccer injuries have demonstrated that up to $80 \%$ of all injuries are experienced by the lower extremities, with the knee and the ankle being the most commonly injured parts of the lower limbs [5].

Kicking motions have been previously linked to the aetiology of soccer specific injuries [6]. Rahnama et al. [7] demonstrated that kicking accounts for $51 \%$ of the movements that have been linked to the cause of soccer injuries Examination of the mechanical demands of atypical soccer indicates that during a 90-minute match, players on average make contact with the ball on 51 occasions, and 26 these were shown to be kicking actions [8]. This shows that the support limb will be loaded on multiple occasions on both dominant and non-dominant sides to produce kicking movements during a soccer game [8].

The majority of published work investigating the biomechanics of instep kicking has focussed on the kicking limb [9, 10, 11], and the supporting limb has received comparatively little attention. Ball [12] examined bilateral differences in ground reaction forces of the support limb during the stance phase of the punt kick. The results showed no significant differences between sides. Kellis et al. [13] investigated knee biomechanics and ground reactions forces of the support limb when kicking from three different approach angles, straight, $45^{\circ}$ and $90^{\circ}$. The $45^{\circ}$ and $90^{\circ}$ kicks were associated with greater medial and posterior forces as well as higher knee flexion, internal rotation, abduction, and adduction velocity.

Patellar tendinopathy is a common and significant syndrome encountered in sports such as soccer where multiple landings are involved [14]. Patellar tendinopathy is associated with pain and clinical dysfunction of the patellar tendon [15]. This condition can severely limit sport participation and performance and has been shown to be career threatening in professional athletes. Patellar tendinopathy is believed to result from repeated loading of the knee extensor mechanism during landing activities [16].

In order to improve performance, soccer players are typically encouraged to develop proficiency in kicking with both limbs [2]. Despite this however, soccer players habitually favour one limb for kicking over the other [17]. The distinctly unilateral nature of instep kicking in soccer may be important clinically and have repercussions with regards to the aetiology of injury. The mechanics of the support limb may be different when kicking with the dominant and non-dominant limbs during instep kicking; however it is still unknown whether these alterations in kicking mechanics modify soccer players' susceptibility to injury.

The aim of the current investigation was to determine whether soccer players exhibit bilateral differences in support limb patellar tendon loads that may provide insight into the aetiology of injury. This study tests the hypothesis that kicking with the non-dominant limb will lead to increased loads experienced by the patellar tendon. 


\section{MATERIAL AND METHODS}

\section{PARTICIPANTS}

Fifteen male soccer players (age $=18.1 \pm 1.2$ years; height $=1.7 \pm 0.1 \mathrm{~m}$; body mass $=73.5 \pm 6.2 \mathrm{~kg}$ ) were examined whilst kicking a stationary soccer ball into a regulation sized goal with both their dominant and non-dominant feet. As all participants were right foot dominant, this meant that the dominant support limb was the left leg and the non-dominant support limb the right leg. The dominant supporting limb was the limb most often in contact with the ground during kicking; this was the left limb in all participants. Participants were academy level soccer players contracted to a professional club in England. Each player trained 4 times per week and had at least 8 years of competitive soccer experience. All were free from lower limb pathology at the time of data collection and provided written informed consent in accordance with the procedures outlined in the declaration of Helsinki. Ethical approval was obtained from the school of Psychology.

\section{PROCEDURE}

Data collection was undertaken in a $22 \mathrm{~m}$ biomechanics laboratory. Instep kicking movements were collected using a ten-camera optoelectric motion analysis system (Qualisys ${ }^{\mathrm{TM}}$ Medical AB, Göteborg, Sweden) operating at $500 \mathrm{~Hz}$. Soccer players were instructed to perform maximal velocity instep kicks with their right (dominant) and left (non-dominant) feet. A $5 \mathrm{~m}$ run-up was allowed with an approach angle of $45^{\circ}$ [18]. The ball was positioned so that it allowed the support foot to land on an embedded piezoelectric force platform (Kistler Instruments, Model 9281CA) which sampled at $1000 \mathrm{~Hz}$. This allowed joint moments of the stance limb to be quantified. Kinematics and force information were collected synchronously using an analogue to digital interface board. A total of 5 successful trials were recorded for both the dominant and non-dominant sides. One minute of rest was allowed between trials. The order in which the participants performed kicks with their dominant and non-dominant sides was randomized. A trial was considered to be successful if the ball entered the goal and the participants' stance foot made contact with the force platform; on average participants required $6.7 \pm 1.3$ attempts to complete 5 trials.

The calibrated anatomical systems technique (CAST) was used to quantify joint kinematics [19]. A total of 10 retroreflective markers (19 $\mathrm{mm}$ in diameter) were positioned bilaterally onto the medial and lateral malleolus, medial and lateral epicondyle of the femur and greater trochanter. Technical tracking clusters, which consisted of four $19 \mathrm{~mm}$ in diameter spherical reflective markers mounted to a thin sheath of lightweight carbon fibre, were positioned bilaterally onto the thigh and the shank. A static trial was captured with participants in the anatomical position, which allowed the thigh and shank segments to be delineated and tracked (Figure 1). Following acquisition of the static trial markers not used for tracking, the segments during motion, i.e. femoral epicondyle and malleolus markers were removed prior to the collection of dynamic information. All soccer players were provided with the same footwear; Adidas Kaiser 5 team astro trainers in sizes 6-9 UK. 


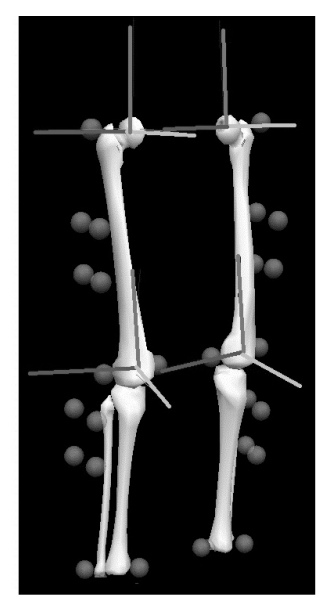

Fig. 1. Lower extremity model and markers

\section{DATA PROCESSING}

Ground reaction force and marker trajectories were filtered at 50 and $12 \mathrm{~Hz}$ using a low pass Butterworth 4th order zero-lag filter and analysed using Visual 3D (C-Motion, Germantown, MD, USA). Kinematics of the knee were quantified using an $\mathrm{XYZ}$ cardan sequence of rotations (where $\mathrm{X}=$ sagittal plane, $\mathrm{Y}=$ coronal plane, and $\mathrm{Z}=$ transverse plane). Knee kinetic and kinematic curves were normalized to $100 \%$ of the kick phase. Joint moments were computed using Newton-Euler inverse-dynamics. The net joint moments were subsequently normalized to participants' body mass and $(\mathrm{Nm} / \mathrm{kg})$.

To estimate patellar tendon force (PTF), a predictive algorithm was utilized [20]. This model has been used previously to resolve differences in PTF during different sports movements [16, 21]. PTF was determined by dividing the knee extensor moment (KXM) by the estimated patellar tendon moment arm (ptMA). The moment arm was quantified as a function of the sagittal plane knee angle by fitting a $2^{\text {nd }}$-order polynomial curve to the data provided by [22] showing patellar tendon moment arm areas at varying degrees of knee flexion.

$\mathrm{PTF}=\mathrm{KXM} / \mathrm{ptMA}$

PTF values were normalized by dividing by bodyweight (BW). In addition, the PTF loading rate (BW/s) was calculated by dividing PTF by the time taken to reach this value. Finally, approach velocity at the instance of stance limb foot contact was quantified when kicking with the dominant and non-dominant limbs.

\section{STATISTICAL ANALYSES}

To compare differences in patellar tendon loading and approach velocity between the dominant and non-dominant limbs, paired samples t-tests were utilized with significance accepted at $\mathrm{p} \leq 0.05$. Effect sizes were calculated using partial eta ${ }^{2}\left(\mathrm{p \eta}^{2}\right)$. Cohen's suggestion on effect sizes was adopted ( $\operatorname{small}=0.2$; medium $=0.50$; large $=0.80$ ). The Shapiro-Wilk statistic for each condition confirmed that the data were normally distributed. All statistical procedures were conducted using SPSS v22.0 (SPSS Inc., Chicago, IL, USA). 


\section{RESULTS}

Figure 2 and Table 1 present the patellar tendon kinetics as a function of the dominant and non-dominant limbs. The results indicate that there were significant differences in patellar tendon forces as a function of kicking with the dominant and non-dominant limbs.

\section{APPROACH VELOCITY}

Approach velocity did not differ significantly between the dominant (3.31 $\pm 1.01 \mathrm{~m} / \mathrm{s})$ and non-dominant $(3.27 \pm 0.97 \mathrm{~m} / \mathrm{s})$ conditions.

\section{PATELLAR TENDON KINETICS}
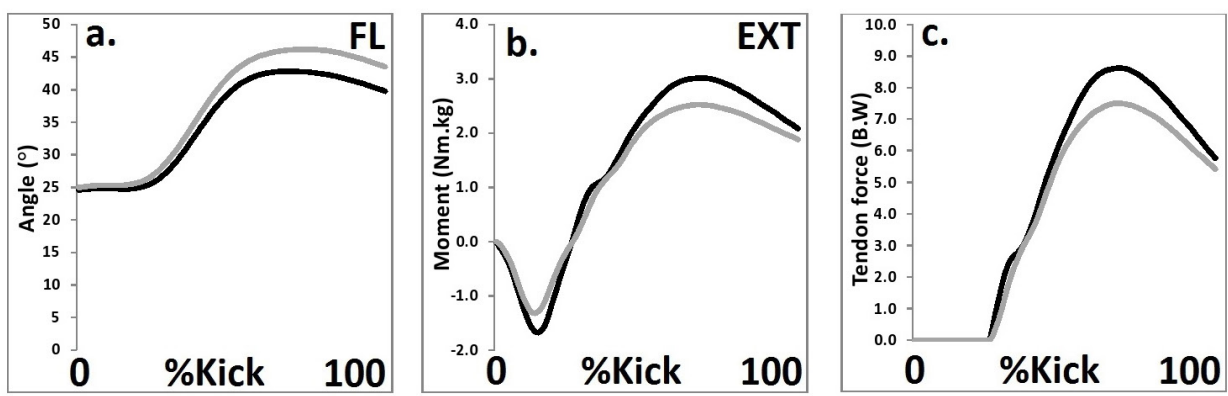

Fig. 2. Knee kinetics and kinematics when kicking with the dominant (grey line) and non-dominant (black line) limbs ( $a=$ knee angle, $b=$ knee moment, $c=$ patellar tendon kinetics) ( $F L=$ flexion, EXT $=$ extension).

Table 1. Patellar tendon kinetics as a function of the dominant and non-dominant limbs

\begin{tabular}{|c|c|c|c|c|c|}
\hline & Dominant & SD & Non-dominant & SD & \\
\hline Peak knee extensor moment (Nm/kg) & 2.48 & 0.56 & 3.18 & 0.67 & $*$ \\
\hline PTF (BW) & 7.34 & 2.61 & 8.62 & 2.94 & $*$ \\
\hline Time to peak force (s) & 0.12 & 0.05 & 0.12 & 0.05 & \\
\hline PTF Load rate (BW/s) & 61.08 & 12.22 & 71.59 & 13.64 & $*$ \\
\hline
\end{tabular}

* = significant difference

The results indicate that peak KXM was significantly $\left(_{(14)}=5.32, \mathrm{p} \leq 0.05\right.$, $\mathrm{p \eta}^{2}=0.48$ ) larger when kicking with the non-dominant limb (Table 1; Figure $2 b$ ). In addition, it was also shown that peak PTF was significantly larger $\left(\mathrm{t}_{(14)}=4.79, \mathrm{p} \leq 0.05, \mathrm{p}^{2}=0.44\right)$ when kicking with the non-dominant limb (Table 1; Figure 2c). Finally it was also demonstrated that the PTF loading rate was significantly larger $\left(\mathrm{t}_{(14)}=4.84, \mathrm{p} \leq 0.05, \mathrm{p}^{2}=0.45\right)$ when using the non-dominant limb (Table 1).

\section{DISCUSSION}

To the authors' knowledge, the present study represents the first comparative examination of the bilateral loads imposed on the patellar tendon during instep kicking. The current work may therefore provide new insight into the aetiology of bilateral pathologies to the support limb in soccer players. 
The first important biomechanical observation from the current study is that the non-dominant limb was associated with significantly increased KXM in relation to the dominant side during instep kicking. This observation supports the observations of Clagg et al. [23], who also demonstrated increases in knee extensor moment when kicking with the non-dominant limb. In support of our hypothesis our findings also demonstrated that PTF was also significantly greater in the non-dominant limb. This finding may have clinical relevance with regards the aetiology of chronic injury development when kicking with the non-dominant limb, as the clinical consensus regarding the development of patellar tendonitis is that the symptoms are a function of excessive tendon loading [24]. Therefore, the current investigation indicates that kicking using the non-dominant limb may place soccer players at increased risk of patellar tendon injury. It is hypothesized that this observation may relate to the reduced knee flexion observed when kicking with the non-dominant limb. Reductions in knee flexion are associated with a shortening of the moment arm of the patellar tendon, which ultimately leads to an increase in the load experienced by the tendon itself [17].

Increases in KXM when using the non-dominant limb indicate that soccer players utilized higher levels of braking torque at the knee. This supports the propositions of Clagg et al. [23] that soccer players are more guarded when kicking with their non-favoured foot, particularly as approach did not differ between the two kicking conditions. Clagg et al. [23] indicate that players utilize a more novice kicking technique when using their non-dominant limb. Therefore, differences in peak KXM may be indicative of a difference in motor control or could also be related to reduced strength in the support limb musculature.

A drawback of the current study is that the sample consisted of only male soccer players. Previous analyses have demonstrated that there are gender differences in lower extremity kinematics [25]. Females have been shown to be at greater risk from injuries at the knee in relation to mal-alignment during periods of loading [26]. There is currently a lack of research investigating the mechanics of in-step kicking in females. It is, therefore, strongly advocated that the current investigation be repeated with a sample of female soccer players. In addition, this work examined the dominant and non-dominant limbs only during maximal kicking. Therefore, the effects of sub-maximal kicking on the loads experienced by the patellar tendon remain unknown. As such, the current analysis should be repeated using sub-maximal kicking trials, which may yield further clinically meaningful observations in relation to the aetiology of patellar tendon pathologies.

A further potential drawback is that PTF was quantified using a mathematical model. This was due to the invasive nature of obtaining direct measures of PTF. Future work should nonetheless seek to develop a patellar tendon model which is more specific to participants' knee morphology. Muscle driven simulations of knee forces using inverse kinematics have improved considerably and have therefore been cited as a useful tool for the quantification of musculoskeletal forces [27]. Musculoskeletal simulations remain relatively new, but with developmental work to improve their accuracy, further advancements in musculoskeletal research are possible. 


\section{CONCLUSIONS}

In conclusion, although previous analyses have comparatively examined the mechanics of kicking with the dominant and non-dominant limbs, the current knowledge concerning the differences in patellar tendon forces between the two modalities is limited. The current investigation addresses this by providing a comparison of patellar tendon forces when running kicking with the dominant and non-dominant feet. The current study shows that patellar tendon kinetics were significantly larger when using the non-dominant limb. This may have clinical implications for the aetiology of bilateral injuries in soccer players and suggests that kicking with the non-dominant limb may place the patellar tendon at increased risk of injury.

\section{ACKNOWLEDGEMENT}

Thanks go to Glen Crook for his technical help and support throughout this work.

\section{REFERENCES}

[1] Lees A, Asai T, Andersen TB, Nunome H, Sterzing T. The biomechanics of kicking in soccer: A review. J Sport Sci. 2010;28:805-817.

[2] Sinclair J, Fewtrell D, Taylor PJ, Atkins S, Bottoms L, Hobbs SJ. Three-dimensional kinematic differences between the preferred and non-preferred limbs during maximal instep soccer kicking. J Sport Sci. 2014;32:1914-1923.

[3] Keller CS, Noyes FR, Buncher CR. The medical aspects of soccer injury epidemiology. Am J Sports Med. 1987;15:230-237.

[4] Junge A, Dvorak J, Graf-Baumann T. Football injuries during the World Cup 2002. Am J Sports Med. 2004;32:23-27.

[5] Fried T, Lloyd GJ. An overview of common soccer injuries. Sports Med. 1992;14:269-275.

[6] Hawkins RD, Hulse MA, Wilkinson C, Hodson A, Gibson M. The association football medical research programme: An audit of injuries in professional football. Brit J Sport Med. 2001;35:43-47.

[7] Rahnama N, Reilly T, Lees A. Injury risk associated with playing actions during competitive soccer. Brit J Sport Med. 2002;36:354-359.

[8] Withers RT, Maricic Z, Wasilewski S, Kelly L. Match analysis of Australian professional soccer players. J Hum Movement Stud. 1982;8:159-176.

[9] Dorge HC, Anderson TB, Sorensen H, Simonsen EB. Biomechanical differences in soccer kicking with the preferred and the non-preferred leg. J Sport Sci. 2002;20:293-299.

[10] Barfield WR, Kirkendall D, Yu, B. Kinematic instep kicking differences between elite female and male soccer players. Journal of Sports Science and Medicine. 2002;3:72-79.

[11] Kellis E, Katis A, Vrabas IS. Effects of an intermittent exercise fatigue protocol on biomechanics of soccer kick performance. Scandinavian Journal of Medicine and Science in Sports. 2006;16:334-344

[12] Ball K. Loading and performance of the support leg in kicking. Journal of Science and Medicine in Sport. 2013;16:455-459.

[13] Kellis E, Katis A, Gissis I. Knee biomechanics of the support leg in soccer kicks from three angles of approach. Med Sci Sport Exer. 2004;336:1017-1028.

[14] Hägglund M, Waldén M, Zwerver J, Ekstrand, J. Epidemiology of patellar tendon injury in elite male soccer players. Brit J Sport Med. 2011;45:324-328.

[15] Rutland M, O’Connell D, Brismée JM, Sizer P, Apte G, O’Connell J. Evidence-supported rehabilitation of patellar tendinopathy. North American Journal of Sports Physical Therapy. 2010;5:166-178.

[16] Lian ØВ, Engebretsen L, Bahr R. Prevalence of Jumper's Knee Among Elite Athletes From Different Sports A Cross-sectional Study. Am J Sports Med. 2005;33:561-567.

[17] Carey DP, Smith G, Smith DT, Shepherd JW, Skriver J, Ord L, Rutland, A. Footedness in world soccer: an analysis of France 98. J Sport Sci. 2001;19:855-864.

[18] Isokawa M, Lees AA. Biomechanical analysis of the instep kick motion in soccer. In T. Reilly, A. Lees, K. Davids, and W. Murphy (Eds.). Proceedings of oral sessions, Science and Football. London: E and FN Spon, 1988: 449-455.

[19] Cappozzo A, Catani F, Leardini A, Benedeti MG, Della CU. Position and orientation in space of bones during movement: Anatomical frame definition and determination. Clin Biomech. 1995;10:171-178.

[20] Janssen I, Steele JR, Munro BJ, Brown NA. Predicting the patellar tendon force generated when landing from a jump. Med Sci Sport Exer. 2013;45:927-934. 
[21] Sinclair J, Bentley I, Kudiersky N, Atkins S, Vincent H. Effects of four different relative loads on knee joint kinetics during the barbell back squat. Journal of Multidisciplinary Engineering Science and Technology. 2014;2:286-290.

[22] Herzog W, Read LJ. Lines of action and moment arms of the major force-carrying structures crossing the human knee joint. J Anat. 1993;182:213-230.

[23] Clagg SE, Warnock A, Thomas JS. Kinetic analyses of maximal effort soccer kicks in female collegiate athletes. Sports Biomech. 2009;8:141-153.

[24] Lavagnino M, Arnoczky SP, Elvin N, Dodds J. Patellar tendon strain is increased the site of the jumper's knee lesion during knee flexion and tendon loading results and cadaveric testing of a computational model. Am J Sports Med. 2008;36:2110-2118.

[25] Barfield WR. Biomechanics of kicking in soccer. Clin Sport Med. 1998;117:711-728.

[26] Robinson RL, Nee RJ. Analysis of hip strength in females seeking physical therapy treatment for unilateral patellofemoral pain syndrome. J Orthop Sport Phys. 2007;37:232-238.

[27] Delp SL, Anderson FC, Arnold AS, et al. Open Sim: open-source software to create and analyze dynamic simulations of movement. IEEE Transactions in Biomedical Engineering. 2007;54:1940-1950.

\section{Cite this article as:}

